\title{
Comparison of errors and stability in FDTD and FVTD
}

\author{
P. Leuchtmann, C. Fumeaux, and D. Baumann \\ Institut für Feldtheorie und Höchstfrequenztechnik, ETH Zürich, CH-8092 Zürich, Switzerland
}

\begin{abstract}
The methods of finite-difference time-domain (FDTD) and finite-volume time-domain (FVTD) are well known techniques for solving electromagnetic problems. While for FDTD a number of commercial codes is available there are only few based on FVTD. However, FVTD has advantages since it allows completely unstructured meshes. This paper 1. deals with the principle errors made in both approaches and 2. gives a theoretical comparison of the (local) stability of an FVTD scheme as opposed to the conventional Yee-scheme commonly used in FDTD. Finally the principles of a new FVTD program is presented. Its capabilities are demonstrated by the field of an antenna array.
\end{abstract}

\section{From field functions to numbers}

In any numerical technique for solving Maxwell's equations the vector-valued $\boldsymbol{r}, t$ (space,time) functions $\boldsymbol{E}(\boldsymbol{r}, t)$ (electric field strength), $\boldsymbol{H}(\boldsymbol{r}, t)$ (magnetic field strength), $\boldsymbol{D}(\boldsymbol{r}, t)$ (displacement density) and $\boldsymbol{B}(\boldsymbol{r}, t)$ (magnetic flux density) are mapped to a finite set of numbers to be processed by the computer. In the well-known Yee-scheme (Yee, 1966; Taflove and Hagness, 2000) field components are taken at particular locations on a cubic grid such that the component values can be seen as "exact" values at the respective locations and the equations applied to these values can be interpreted as second-order finite-difference approximations of Maxwell's equations. E.g., the $z$-component of Maxwell's first equation is $-\frac{\partial}{\partial t} B_{z}(\boldsymbol{r}, t)=\frac{\partial}{\partial x} E_{y}(\boldsymbol{r}, t)-\frac{\partial}{\partial y} E_{x}(\boldsymbol{r}, t)$. This can be approximated to second-order accuracy at the location $(\boldsymbol{r}, t)$ by

$$
\begin{aligned}
& -\frac{B_{z}\left(+\delta_{t}\right)-B_{z}\left(-\delta_{t}\right)}{2 \delta_{t}} \approx \\
& \frac{E_{y}\left(+\delta_{x}\right)-E_{y}\left(-\delta_{x}\right)}{2 \delta_{x}}-\frac{E_{x}\left(+\delta_{y}\right)-E_{x}\left(-\delta_{y}\right)}{2 \delta_{y}}
\end{aligned}
$$

Correspondence to: P. Leuchtmann

(leuchtmann@ifh.ee.ethz.ch) where $\delta_{x}, \delta_{y}, \delta_{z}, \delta_{t}$ are the respective half grid constants and say $E_{y}\left(+\delta_{x}\right)$ is short for $E_{y}\left(x+\delta_{x}, y, z, t\right)$. This means that the approximation of this particular component of Maxwell's equations at the location $(\boldsymbol{r}, t)$ needs only field values being half space- or time-steps apart from this location. Yee's scheme uses different locations for different field components. Fig. 1a shows the spatial location of both the components and the equations. Note that the material equations ( $\boldsymbol{B}=\mu \boldsymbol{H}$ and $\boldsymbol{D}=\varepsilon \boldsymbol{E}$ ) are easily satisfied for each component separately at its own location. The Yee-scheme consists of set $A_{Y}$ : six Maxwellian component Eqs. (1) plus set $B_{Y}$ : six material equations. Set $A_{Y}$ is of second-order accuracy, set $B_{Y}$ is exactly satisfied.

In the finite integration technique (FIT) (Weiland, 1977) Maxwell's first equation in integral form $\left(-\frac{\partial}{\partial t} \iint_{A} \boldsymbol{B} \cdot \boldsymbol{d} \boldsymbol{A}=\right.$ $\left.\oint_{\partial A} \boldsymbol{E} \cdot \boldsymbol{d} \boldsymbol{s}\right)$ is applied to the green rectangle shown in Fig. 1(b).

$$
\begin{aligned}
& -\frac{\partial}{\partial t}\left\langle B_{z}\right\rangle_{\mathrm{A}} \cdot 2 \delta_{x} \cdot 2 \delta_{y}=\left\langle E_{y}\right\rangle_{\mathrm{L}}^{+\delta_{x}} \cdot 2 \delta_{y}-\left\langle E_{y}\right\rangle_{\mathrm{L}}^{-\delta_{x}} \cdot 2 \delta_{y}- \\
& \left(\left\langle E_{x}\right\rangle_{\mathrm{L}}^{+\delta_{y}} \cdot 2 \delta_{x}-\left\langle E_{x}\right\rangle_{\mathrm{L}}^{-\delta_{y}} \cdot 2 \delta_{x}\right)
\end{aligned}
$$

Thereby $\langle.\rangle_{\mathrm{L}}^{\delta}$ means a line mean value along the respective side of the rectangle and $\langle.\rangle_{\mathrm{A}}$ means the surface mean value. After integrating the whole Eq. (2) along the time interval from $t-\delta_{t}$ to $t+\delta_{t}$ and then dividing it by $8 \delta_{x} \delta_{y} \delta_{t}$ we obtain time mean values (denoted by overbars) at the right-hand side and surface mean values evaluated at the boundary of the time interval at the left-hand side:

$$
\begin{aligned}
& -\frac{\left\langle B_{z}\right\rangle_{\mathrm{A}}\left(+\delta_{t}\right)-\left\langle B_{z}\right\rangle_{\mathrm{A}}\left(-\delta_{t}\right)}{2 \delta_{t}}= \\
& \frac{\left\langle\overline{E_{y}}\right\rangle_{\mathrm{L}}^{+\delta_{x}}-\left\langle\overline{E_{y}}\right\rangle_{\mathrm{L}}^{-\delta_{x}}}{2 \delta_{x}}-\frac{\left\langle\overline{E_{x}}\right\rangle_{\mathrm{L}}^{+\delta_{y}}-\left\langle\overline{E_{x}}\right\rangle_{\mathrm{L}}^{-\delta_{y}}}{2 \delta_{y}}
\end{aligned}
$$

Though formally identical to the approximative relation (1) this is an exact equation. As in Yee's scheme the whole set of Maxwell's eqations can be derived. However, the material equations are now approximatively satisfied since, e.g., the line-time-mean value $\left\langle\overline{H_{z}}\right\rangle_{\mathrm{L}}$ is related to the surface mean 

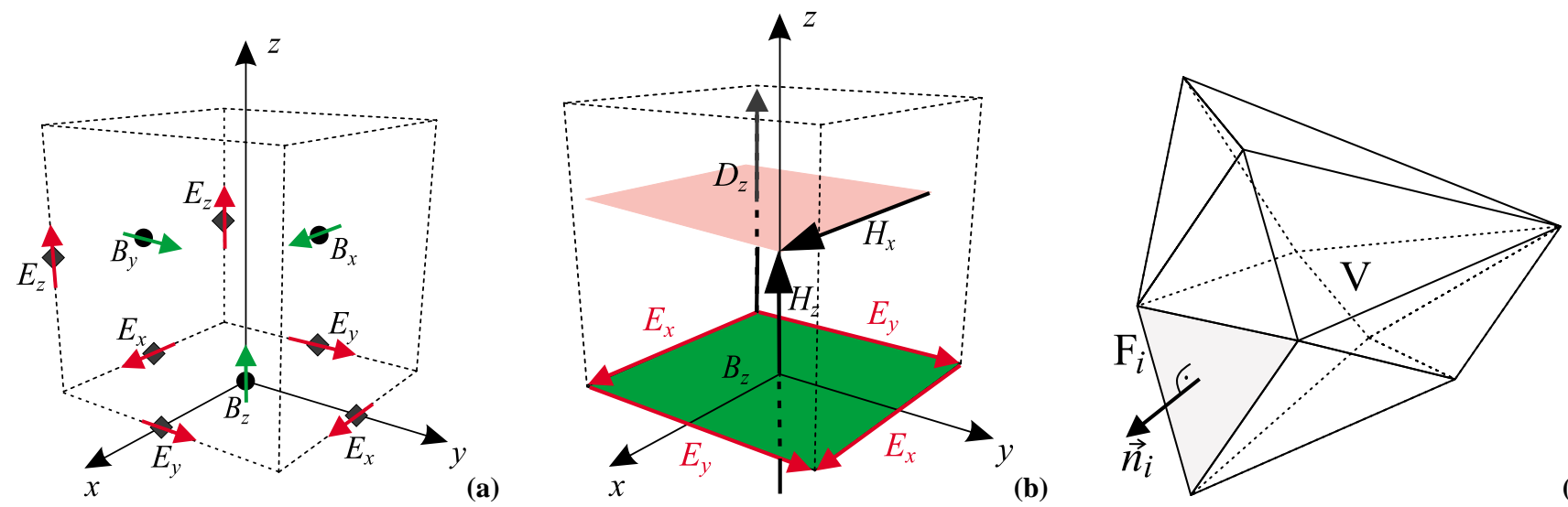

(c)

Fig. 1. The well-known Yee-scheme (a) places different field components at different locations. We use • for magnetic components and for electric components. The respective values are used to form second-order accurate difference equations. The FIT-approach (b) uses mean values ( $B$ - and $D$-components: surface mean values; $E$ - and $H$-components: line-mean values). In FVTD (c) volume-mean values $(\boldsymbol{B}$ - and $\boldsymbol{D}$-vectors) and face-mean values ( $\boldsymbol{E}$ - and $\boldsymbol{H}$-vectors) are used.

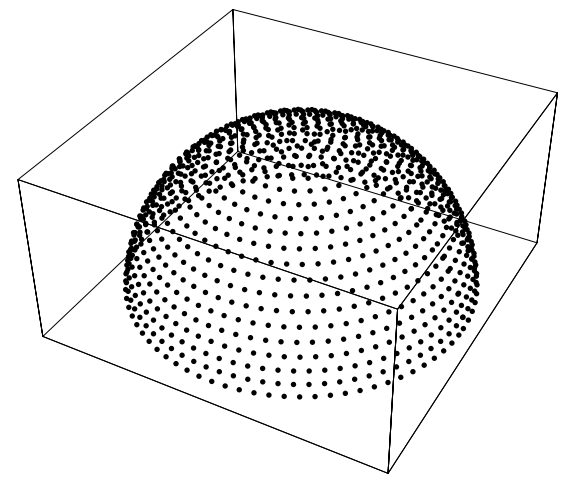

Fig. 2. The picture shows 988 wave vector directions (from the sphere's center). The numerical schemes are tested using plane waves propagating in the respective directions.

value $\left\langle B_{z}\right\rangle_{\mathrm{A}}$ which is a value at a fixed time. The FIT-scheme consists of set $\mathrm{A}_{\mathrm{F}}$ : six Maxwellian component equations plus set $\mathrm{B}_{\mathrm{F}}$ : six material equations. Set $\mathrm{A}_{\mathrm{F}}$ is exactly satisfied while set $\mathrm{B}_{\mathrm{F}}$ is approximate.

In the finite-volume time-domain (FVTD) approach Maxwell's equations can also be integrated over a volume $\mathrm{V}$ rather than a surface $\mathrm{A}$ as done in the FIT-derivation in the previous section. We assume a polyhedron $\mathrm{V}$ with $N$ surfaces $\mathrm{F}_{i}$ forming the boundary $\partial \mathrm{V}$ of $\mathrm{V}$ (see Fig. 1c) and find, e.g.,

$$
-\frac{\partial}{\partial t} \underbrace{\iiint_{\mathrm{V}} \boldsymbol{B} d V}_{V \cdot\langle\boldsymbol{B}\rangle_{\mathrm{V}}}=-\oiint_{\partial \mathrm{V}} \boldsymbol{E} \times \boldsymbol{d} \boldsymbol{A}=\sum_{i=1}^{N} \boldsymbol{n}_{i} \times \underbrace{\iint_{\mathrm{F}_{i}} \boldsymbol{E} d A}_{F_{i} \cdot\langle\boldsymbol{E}\rangle_{\mathrm{F}_{i}}},
$$

where $\langle\boldsymbol{E}\rangle_{\mathrm{F}_{i}}$ and $\langle\boldsymbol{B}\rangle_{\mathrm{V}}$ are the time-dependent mean values of the vector functions $\boldsymbol{E}$ (or $\boldsymbol{B}$ ) on the polyhedrons $i$-th face $\mathrm{F}_{i}$ (or the volume respectively) and $\boldsymbol{n}_{i}$ is the respective outer normal unit vector. Hence Eq. (4) is an exact relation between time dependent mean values. If Eq. (4) is integrated over time an exact vector-valued equation somehow similar to Eq. (3) is found:

$$
-\frac{\langle\boldsymbol{B}\rangle_{\mathrm{V}}\left(+\delta_{t}\right)-\langle\boldsymbol{B}\rangle_{\mathrm{V}}\left(-\delta_{t}\right)}{2 \delta_{t}}=\frac{1}{V} \sum_{i=1}^{N} F_{i}\left(\boldsymbol{n}_{i} \times\langle\overline{\boldsymbol{E}}\rangle_{\mathrm{F}_{i}}\right) .
$$

Using Maxwell's second equation another exact vector equation involving $\langle\boldsymbol{D}\rangle_{\mathrm{V}}$ and $\langle\overline{\boldsymbol{H}}\rangle_{\mathrm{F}_{i}}$ values is obtained.

Concerning time the face mean values are "older" than the newest volume mean value at the left hand side. "Newer" face mean values might be obtained by the extrapolation

$$
\begin{aligned}
& \langle\overline{\boldsymbol{E}}\rangle_{\mathrm{F}}\left(t+2 \delta_{\mathrm{t}}\right) \approx 2\langle\overline{\boldsymbol{E}}\rangle_{\mathrm{F}}\left(t+\delta_{\mathrm{t}}\right)-\langle\overline{\boldsymbol{E}}\rangle_{\mathrm{F}}(t), \\
& \langle\overline{\boldsymbol{H}}\rangle_{\mathrm{F}}\left(t+2 \delta_{\mathrm{t}}\right) \approx 2\langle\overline{\boldsymbol{H}}\rangle_{\mathrm{F}}\left(t+\delta_{\mathrm{t}}\right)-\langle\overline{\boldsymbol{H}}\rangle_{\mathrm{F}}(t) .
\end{aligned}
$$

Since each face has two sides one can set up further relations, e.g.

$$
\begin{aligned}
& \langle\overline{\boldsymbol{H}}\rangle_{\mathrm{F}} \approx \frac{w_{\mathrm{l}}}{\mu_{1}}\langle\boldsymbol{B}\rangle_{\mathrm{V}_{\mathrm{l}}}+\frac{w_{\mathrm{r}}}{\mu_{\mathrm{r}}}\langle\boldsymbol{B}\rangle_{\mathrm{V}_{\mathrm{r}}} \\
& \langle\overline{\boldsymbol{E}}\rangle_{\mathrm{F}} \approx \frac{w_{1}}{\varepsilon_{1}}\langle\boldsymbol{D}\rangle_{\mathrm{V}_{\mathrm{l}}}+\frac{w_{\mathrm{r}}}{\varepsilon_{\mathrm{r}}}\langle\boldsymbol{D}\rangle_{\mathrm{V}_{\mathrm{r}}} \\
& \text { or even } \begin{array}{l}
\langle\overline{\boldsymbol{H}}\rangle_{\mathrm{F}} \approx f\left(\langle\boldsymbol{B}\rangle_{\mathrm{V}_{\mathrm{l}}},\langle\boldsymbol{B}\rangle_{\mathrm{V}_{\mathrm{r}}},\langle\boldsymbol{D}\rangle_{\mathrm{V}_{\mathrm{l}}},\langle\boldsymbol{D}\rangle_{\mathrm{V}_{\mathrm{r}}}\right) \\
\overline{\langle\boldsymbol{E}}\rangle_{\mathrm{F}} \approx g\left(\langle\boldsymbol{B}\rangle_{\mathrm{V}_{\mathrm{l}}},\langle\boldsymbol{B}\rangle_{\mathrm{V}_{\mathrm{r}}},\langle\boldsymbol{D}\rangle_{\mathrm{V}_{\mathrm{l}}},\langle\boldsymbol{D}\rangle_{\mathrm{V}_{\mathrm{r}}}\right)
\end{array}
\end{aligned}
$$

where the indices $r$ and 1 stand for "left" and "right" (of the face F) respectively. The quantities $w_{\mathrm{r}, 1}$ are some weights due to geometry. In a simple symmetrical case (two identical cells) it is $w_{\mathrm{r}}=w_{1}=\frac{1}{2}$. In the more complicated case, $f$ and $g$ are some functions yet to be specified. Regardless of the particular choices both Eqs. (6) and (7) are approximate relations.

FVTD ends up with an exact set $A_{V}$ of six scalar Eq. (5) and a second set $\mathrm{B}_{\mathrm{V}}$ - formed with Eqs. (6) and (7) - of approximate relations.

As it is well known both sets of equations are combined to an update scheme which allows it to compute "new" values (written in $n$-dimensional vector $[V]_{\text {new }}$ ) from the "old" values $[V]_{\text {old }}$. The respective scheme can be formally written 

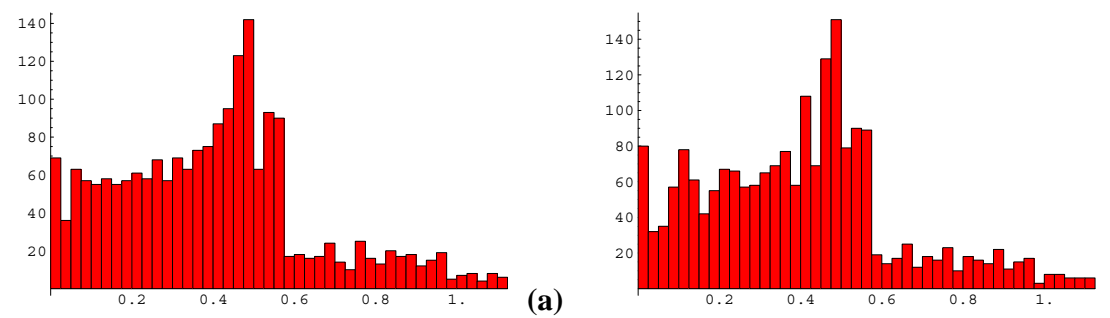

(b)

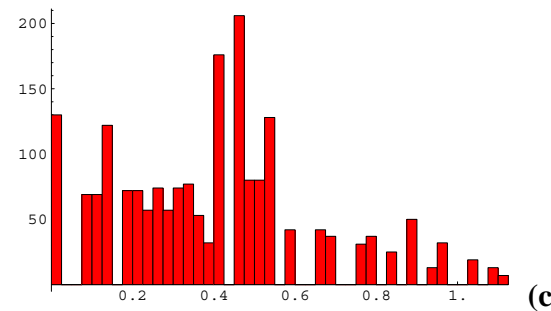

Fig. 3. The histograms of the FIT-errors according to Eq. (10) for $\frac{\lambda}{2 \delta_{\xi}}=14 \ldots 15$ (cells per wave length). Maximum values are around $1 \%$. From left to right $\eta_{\text {FITx }}, \eta_{\text {FITy }}$ and $\eta_{\text {FITz }}$ are shown.

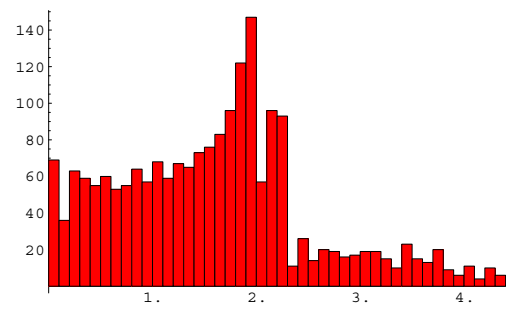

(a)
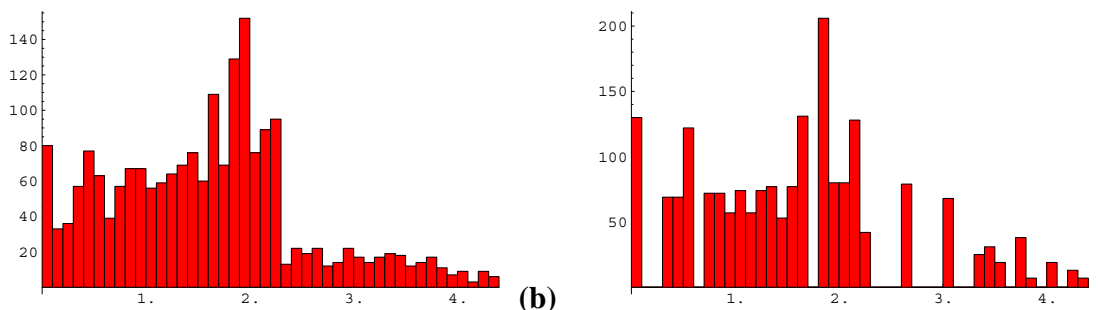

(c)

Fig. 4. Histograms of the FVTD-errors according to Eq. (11) for $\frac{\lambda}{2 \delta_{\xi}}=14 \ldots 15$ (cells per wave length). Maximum values are around $4 \%$ which is four times more than in FIT. This higher value is not a principle drawback of FVTD but mainly due to our simple choice in Eqs. (6) and (7): essentially a first order scheme both in space and time. From left to right $\eta_{\text {FVTDx }}, \eta_{\text {FVTDy }}$ and $\eta_{\text {FVTDz }}$ are shown.

with a large matrix $\mathbf{M}_{\text {method }}$ where "method" is one out of FDTD, FIT and FVTD:

$[V]_{\text {new }}=\mathbf{M}_{\text {method }} \cdot[V]_{\text {old }}$.

Two questions can be posed:

Accuracy: "To what degree are the approximate equations wrong in each case?"

Stability: "Under what conditions are the respective schemes numerically stable?"

Since FDTD and FIT differ only in the interpretation of the numbers but lead to formally the same equations we treat only the FIT.

\section{The definition of the errors and their values}

The accuracy depends on several things such as structure and dimension of the mesh but also on the actual field. We define 988 test cases of the actual field: plane waves propagating in different directions. A single plane wave in a homogeneous medium is described by

$\boldsymbol{D}(\boldsymbol{r}, t) / \varepsilon=\boldsymbol{E}(\boldsymbol{r}, t)=\boldsymbol{E}_{0} \cdot \cos (\omega t-\boldsymbol{\kappa} \cdot \boldsymbol{r})$

$\boldsymbol{B}(\boldsymbol{r}, t) / \mu=\boldsymbol{H}(\boldsymbol{r}, t)=\boldsymbol{H}_{0} \cdot \cos (\omega t-\boldsymbol{\kappa} \cdot \boldsymbol{r})$

with $\boldsymbol{H}_{0}=\frac{1}{\omega \mu} \boldsymbol{\kappa} \times \boldsymbol{E}_{0}, \boldsymbol{\kappa} \cdot \boldsymbol{E}_{0}=0, \boldsymbol{\kappa} \cdot \boldsymbol{\kappa}=\omega^{2} \mu \varepsilon$.

The directions of $\boldsymbol{\kappa}:=\left(\kappa_{x}, \kappa_{y}, \kappa_{z}\right)$ and $\boldsymbol{E}_{0}$ are free beside these restrictions.

Given one of these test fields $[V]_{\text {old }}$ is computed for both methods in the respective way (space and/or time mean values) and also the respective exact $[V]_{\text {new,ex }}$. A slightly dif- ferent value for $[V]_{\text {new }}$ is obtained by using Eq. (8). A comparison of $[V]_{\text {new,ex }}$ and $[V]_{\text {new }}$ delivers the respective error.

In order to keep the amount of computation within reasonable limits we restrict the error analysis to the approximative equations and use typical discretisation lengths $\delta_{\xi}$ (fractions of the wavelength $\lambda=\frac{2 \pi}{|\kappa|}$ ).

In FIT the material equations are approximate relations, e.g., $\left\langle D_{\xi}\right\rangle_{\mathrm{A}} \approx \varepsilon\left\langle\overline{E_{\xi}}\right\rangle_{\mathrm{L}}$. From this we derive the error $r_{\mathrm{Ex}}:=\varepsilon\left\langle\overline{E_{\xi}}\right\rangle_{\mathrm{L}} /\left\langle D_{\xi}\right\rangle_{\mathrm{A}} \stackrel{!}{=} 1$. Introducing the expressions given in Eq. (9) and evaluating the mean values by analytical integration we find a value different from 1 for the respective exact ratio:

$\tilde{r}_{\mathrm{Ex}}=\frac{\operatorname{Si}\left(\kappa_{x} \delta_{x}\right) \cdot \operatorname{Si}\left(\kappa_{y} \delta_{y}\right)}{\operatorname{Si}\left(\kappa_{z} \delta_{z}\right) \cdot \operatorname{Si}\left(\omega \delta_{t}\right)} \Rightarrow \eta_{\mathrm{FITx}}:=\left|\tilde{r}_{\mathrm{Ex}}-1\right| \cdot 100 \%$

with $\operatorname{Si}(x):=\frac{\sin x}{x}$. The histograms of these error values evaluated for $\delta_{x}=\delta_{y}=\delta_{z}=\sqrt{\frac{3}{\mu \varepsilon}} \delta_{t}$ and all wave directions shown in Fig. 2 are given in Figs. 3 and 4.

In order to obtain a reasonable basis of comparison we use a regular cubic grid (with $2 \delta_{x} \cdot 2 \delta_{y} \cdot 2 \delta_{z}$-cubes) also in the FVTD case and make the aforementioned simple choices in Eqs. (6) and (7). Moreover we can set $w_{1}=w_{2}=\frac{1}{2}$. Introducing the expressions from Eq. (9) and performing the integrations according to the definitions in the FVTD-formulae we finally obtain for the update equation related to a face oriented in $\xi$-direction

$\eta_{\mathrm{FVTD} \xi}:=2\left|\frac{\operatorname{Si}\left(2 \kappa \xi \delta_{\xi}\right)-\operatorname{Si}\left(2 \omega \delta_{t}\right)}{\operatorname{Si}\left(\omega \delta_{t}\right)}\right| \cdot 100 \%$

The respective histograms of these errors are given in Fig. 4. 


\section{The stability}

The time iterative scheme is a repeated application of Eq. (8). This means that the matrix $\mathbf{M}_{\text {method }}$ should not have any eigenvalue $\lambda$ with $|\lambda|>1$. For a full problem the dimension of $\mathbf{M}_{\text {method }}$ is very large and the respective search for all eigenvalues would be extremely expensive. However, there are special numerical schemes (e.g., the Arnoldi scheme) which find the largest eigenvalue within still reasonable time. In this work we do not follow that way but reduce the number of variables (and with it the dimension of $\mathbf{M}_{\text {method }}$ ) by defining a local stability by focusing on a single cell. Considering the update scheme for a particular value (e.g., by solving Eq. 3 or 5 for the latest $B$-value) we find that for computing all "new" values of a single cell the number of the required "old" values is always larger than the number of "new" values. This simply reflects the fact that "old" values from the neighbour cells are also involved. The respective 'local' update equation would have a small but rectangular matrix $\mathbf{M}_{\text {local }}$. This matrix can be reduced to a quadratic matrix by applying a spatial Fourier transformation to the "old" values. In this case any "old" value can be written as $v_{\text {old }, 0} \cdot e^{j\left(\kappa_{x} \tilde{x}+\kappa_{y} \tilde{y}+\kappa_{z} \tilde{z}\right)}$ where $v_{\text {old, } 0}$ is the value in the cell's center, $(\tilde{x}, \tilde{y}, \tilde{z})$ denotes the displacement and $\kappa=\left(\kappa_{x}, \kappa_{y}, \kappa_{z}\right)$ is the vector of the spatial Fourier frequencies. In particular values required from outside the cell are related to the respective values inside the cell by a simple multiplication with the respective dislocation factor.

The restriction of the stability analysis to a single Fourier term is sufficient if stability is proofed for any Fourier term. This can be deduced from Parseval's theorem: the sum of all Fourier terms (which is the true field) remains stable.

Note that in the rectangular grid neighbour values are simply multiplied by $e^{ \pm j \kappa_{\xi} \delta_{\xi}}$ which remains true even for line-, surface- and volume mean values. Assuming a homogeneous material in and around the cell in FIT/FDTD the $(6 \times 6)$ matrix $\mathbf{M}$ can be written as

$\left[\begin{array}{c}\boldsymbol{H} \\ \boldsymbol{E}\end{array}\right]_{\text {new }}=\underbrace{\left[\begin{array}{cc}\mathbb{U}+\frac{1}{\mu \varepsilon} \mathbf{M}_{E} \mathbf{M}_{H} & \frac{1}{\mu} \mathbf{M}_{E} \\ \frac{1}{\varepsilon} \mathbf{M}_{H} & \mathbb{U}\end{array}\right]}_{\mathbf{M}} \cdot\left[\begin{array}{c}\boldsymbol{H} \\ \boldsymbol{E}\end{array}\right]_{\mathrm{old}}$

with

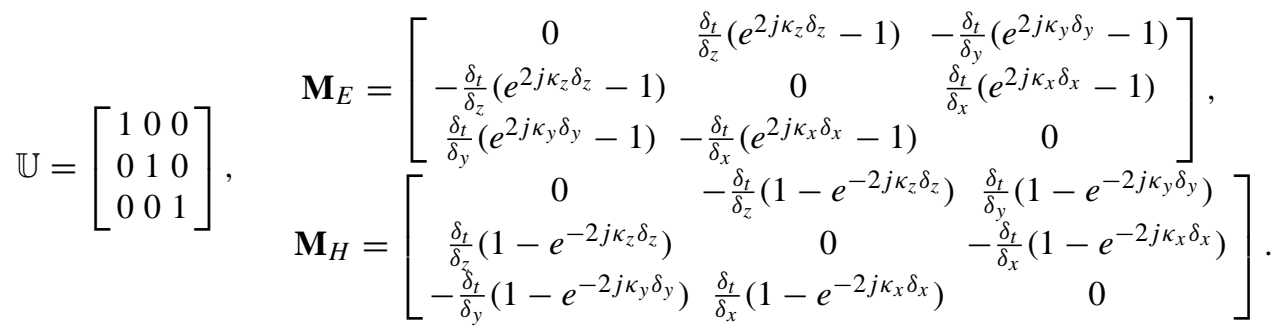

The eigenvalues' amount of $\mathbf{M}$ does not exceed 1 if and only if

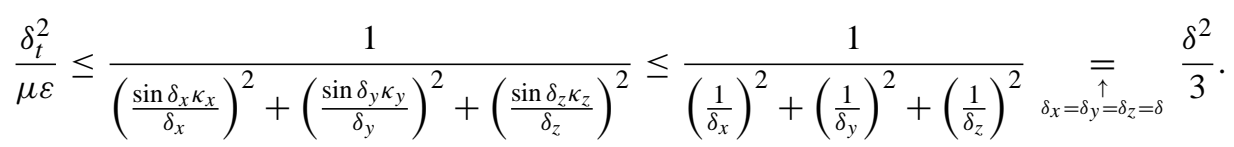

This is the well-known Courant limit.

In FVTD there are 24 scalar variables per cell: 3 face mean values $\boldsymbol{E}_{1,2,3}$ and $\boldsymbol{H}_{1,2,3}$ plus volume mean values of $\boldsymbol{B}$ and $\boldsymbol{D}$. The scheme can be written as

$\left[\begin{array}{llllllll}\boldsymbol{E}_{1} & \boldsymbol{E}_{2} & \boldsymbol{E}_{3} & \boldsymbol{H}_{1} & \boldsymbol{H}_{2} & \boldsymbol{H}_{3} & \boldsymbol{B} & \boldsymbol{D}\end{array}\right]_{\text {new }}^{\mathrm{T}}=\mathbf{M} \cdot\left[\begin{array}{llllllll}\boldsymbol{E}_{1} & \boldsymbol{E}_{2} & \boldsymbol{E}_{3} & \boldsymbol{H}_{1} & \boldsymbol{H}_{2} & \boldsymbol{H}_{3} & \boldsymbol{B} & \boldsymbol{D}\end{array}\right]_{\mathrm{old}}^{\mathrm{T}}$

with the $24 \times 24$-matrix

$\mathbf{M}=\left[\begin{array}{cccccccc}-\mathbb{U} & \mathbf{0} & \mathbf{0} & \frac{1+\alpha_{x}}{\varepsilon} \mathbf{A}_{x} & \frac{1+\alpha_{x}}{\varepsilon} \mathbf{A}_{y} & \frac{1+\alpha_{x}}{\varepsilon} \mathbf{A}_{z} & \mathbf{0} & \frac{1+\alpha_{x}}{\varepsilon} \mathbb{U} \\ \mathbf{0} & -\mathbb{U} & \mathbf{0} & \frac{1+\alpha_{y}}{\varepsilon} \mathbf{A}_{x} & \frac{1+\alpha_{y}}{\varepsilon} \mathbf{A}_{y} & \frac{1+\alpha_{y}}{\varepsilon} \mathbf{A}_{z} & \mathbf{0} & \frac{1+\alpha_{y}}{\varepsilon} \mathbb{U} \\ \mathbf{0} & \mathbf{0} & -\mathbb{U} & \frac{1+\alpha_{z}}{\varepsilon} \mathbf{A}_{x} & \frac{1+\alpha_{z}}{\varepsilon} \mathbf{A}_{y} & \frac{1+\alpha_{z}}{\varepsilon} \mathbf{A}_{z} & \mathbf{0} & \frac{1+\alpha_{z}}{\varepsilon} \mathbb{U} \\ -\frac{1+\alpha_{x}}{\mu} \mathbf{A}_{x} & -\frac{1+\alpha_{x}}{\mu} \mathbf{A}_{y} & -\frac{1+\alpha_{x}}{\mu} \mathbf{A}_{z} & -\mathbb{U} & \mathbf{0} & \mathbf{0} & \frac{1+\alpha_{x}}{\mu} \mathbb{U} & \mathbf{0} \\ -\frac{1+\alpha_{y}}{\mu} \mathbf{A}_{x} & -\frac{1+\alpha_{y}}{\mu} \mathbf{A}_{y} & -\frac{1+\alpha_{y}}{\mu} \mathbf{A}_{z} & \mathbf{0} & -\mathbb{U} & \mathbf{0} & \frac{1+\alpha_{y}}{\mu} \mathbb{U} & \mathbf{0} \\ -\frac{1+\alpha_{z}}{\mu} \mathbf{A}_{x} & -\frac{1+\alpha_{z}}{\mu} \mathbf{A}_{y} & -\frac{1+\alpha_{z}}{\mu} \mathbf{A}_{z} & \mathbf{0} & \mathbf{0} & -\mathbb{U} & \frac{1+\alpha_{z}}{\mu} \mathbb{U} & \mathbf{0} \\ -\mathbf{A}_{x} & -\mathbf{A}_{y} & -\mathbf{A}_{z} & \mathbf{0} & \mathbf{0} & \mathbf{0} & \mathbb{U} & \mathbf{0} \\ \mathbf{0} & \mathbf{0} & \mathbf{0} & \mathbf{A}_{x} & \mathbf{A}_{y} & \mathbf{A}_{z} & \mathbf{0} & \mathbb{U}\end{array}\right]$ 
where 0 stands for a 3-by-3 zero-matrix, $\alpha_{x}:=e^{j \kappa_{x} \delta_{x}}, \alpha_{y}:=e^{j \kappa_{y} \delta_{y}}, \alpha_{z}:=e^{j \kappa_{z} \delta_{z}}$ and

$\mathbf{A}_{\xi}=\frac{\delta_{t}}{\delta_{\xi}}\left(1-1 / \alpha_{\xi}\right) \mathbf{K}_{\xi}, \quad \mathbf{K}_{x}=\left[\begin{array}{ccc}0 & 0 & 0 \\ 0 & 0 & -1 \\ 0 & 1 & 0\end{array}\right], \quad \mathbf{K}_{y}=\left[\begin{array}{ccc}0 & 0 & 1 \\ 0 & 0 & 0 \\ -1 & 0 & 0\end{array}\right], \quad \mathbf{K}_{z}=\left[\begin{array}{ccc}0 & -1 & 0 \\ 1 & 0 & 0 \\ 0 & 0 & 0\end{array}\right]$.

Evaluating the eigenvalues of $\mathbf{M}$ yields

$-1,+1, \pm \sqrt{1-\gamma \frac{\delta_{t}^{2}}{\mu \varepsilon} \pm \sqrt{\left(1-\gamma \frac{\delta_{t}^{2}}{\mu \varepsilon}\right)^{2}-1}} \quad$ with $\quad \gamma=\frac{1-\cos 2 \kappa_{x} \delta_{x}}{\delta_{x}^{2}}+\frac{1-\cos 2 \kappa_{y} \delta_{y}}{\delta_{y}^{2}}+\frac{1-\cos 2 \kappa_{z} \delta_{z}}{\delta_{z}^{2}}$

We find that for $\gamma \delta_{\mathrm{t}}^{2} /(\mu \varepsilon) \leq 2$ all eigenvalues are unimodular complex numbers while for $\gamma \delta_{\mathrm{t}}^{2} /(\mu \varepsilon)>2$, there are eigenvalues with an absolute value being larger than one. A stability-criterion is therefore

$\frac{1}{\delta_{x}^{2}}+\frac{1}{\delta_{y}^{2}}+\frac{1}{\delta_{z}^{2}} \leq \frac{\mu \varepsilon}{\delta_{t}^{2}}$

This is exactly the same as Eq. (14) in FIT/FDTD!

\section{The FVTD program}

A FVTD program is developed in parallel to this theoretical study. To take advantage of the geometrical flexibility of the method, the FVTD algorithm is applied in an unstructured tetrahedral mesh. This type of mesh permits a conformal meshing of complicated geometries including, e.g. curved or oblique surfaces.

The basic FVTD Eq. (4) is numerically integrated in each cell of the mesh in a time-stepping iteration. The approximate relations of the type Eq. (7) are implemented us- ing the following approach: For each face of the cell only the field components tangential to the face are considered (plane-wave ansatz) and the fields are split into incoming and outgoing contributions. Second-order accuracy in space is achieved using the MUSCL approach (monotonic upwind scheme for conservation laws (Bonnet et al., 1999)) that interpolates volume values (assumed located in the barycenter of the cell) to face centers using estimated gradients.

When using second-order accurate schemes in space, the first-order time-stepping scheme of the left side of Eq. (5) is advantageously replaced by the second-order predictor-

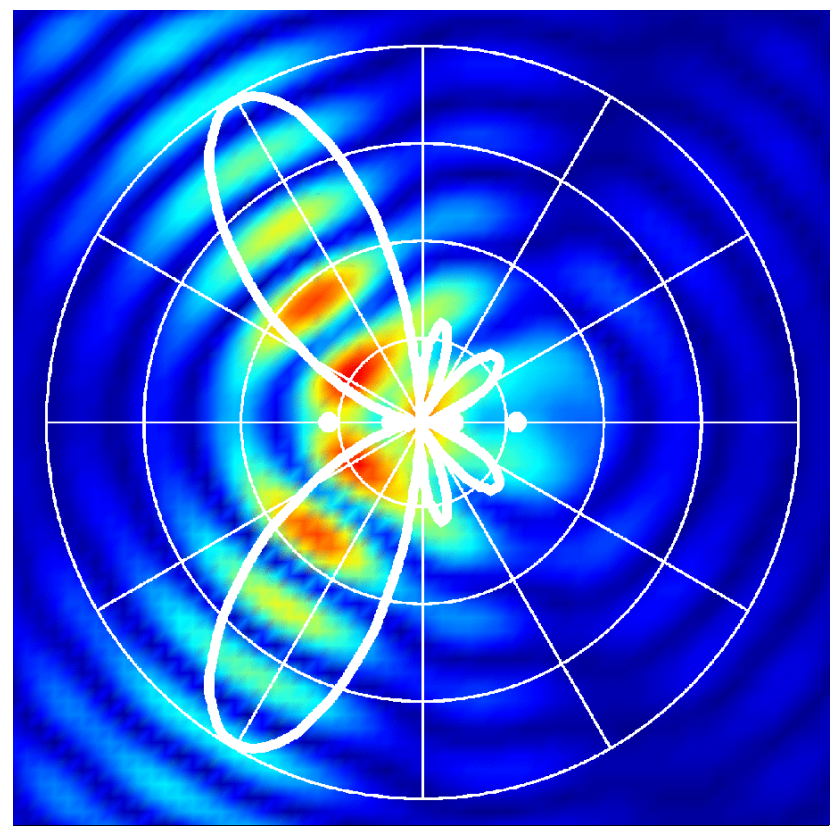

Fig. 5. A four element linear array of Hertzian dipoles (white dots on the horizontal axis). The near-zone E-field (magnitude distribution) is shown. It has been computed with the FVTD method. The array is steered toward $30^{\circ}$ off broadside (= vertical direction). The respective far-field pattern is superimposed in white. 
corrector Lax-Wendroff scheme (Bonnet et al., 1999). This scheme permits as alternative to Eq. (6) to obtain "newer" face mean values in the numerical estimation of Eq. (5). The resulting implemented algorithm is consequently secondorder accurate both in space and time. Absorbing boundary conditions of the Silver-Müller type or Engquist-Majda type are applied to the outer boundary of the computational domain.

Figure 5 shows the near-field distribution of a linear array of four elementary dipoles with half-wave spacing. The plane of observation is perpendicular to the dipoles and located slightly above them. This example validates the FVTD method for EM simulations. The modeling of complicated structures requires only a geometrical definition and meshing of the structure but no change in the FVTD algorithm. Application of the FVTD method to more complicated examples is in progress.

\section{Conclusions}

Common numerical schemes may involve significant errors in particular equations. The FVTD-schemes have many de- grees of freedom. The simple scheme treated here leads to unacceptably high errors. However, it is expected that a more sophisticated scheme delivers much lower errors.

\section{References}

Yee, K. S.: Numerical solution of initial boundary value problems involving maxwell's equations in isotropic media, IEEE Transactions on Antennas and Propagation, 14(3): 302-307, 1966.

Taflove, A. and Hagness, S. C.: Computational Electrodynamics - The finite-difference time-domain method, Artech House, Boston, 2nd edition, 2000.

Weiland, T.: A discretization method for the solution of maxwell's equations for six-component fields, Electronics and Communications AEÜ, 31: 116-120, 1977.

Bonnet, P., Ferrieres, X., Michielsen, B. L., Klotz, P., and Roumiguières, J. L.: Time domain electromagnetics (editor: S.M. Rao), chapter Finite-volume time domain method, Recent Research Developments Series, Academic Press, San Diego, 1999. 